\title{
AMP-activated protein kinase family member 5 is an independent prognostic indicator of pancreatic adenocarcinoma: A study based on The Cancer Genome Atlas
}

\author{
HAOKAI XU ${ }^{1}$, JIAYAN MAO ${ }^{2}$, XIAODAN YANG ${ }^{3}$, FEI CHEN $^{3}$, ZHENGWEI SONG $^{3}$, \\ JIANGUO FEI ${ }^{3}$, WEI CHEN ${ }^{2}$, ZHENGXIANG ZHONG ${ }^{1}$ And XIAOGUANG WANG ${ }^{3}$ \\ ${ }^{1}$ Faculty of Graduate Studies, Bengbu Medical College, Bengbu, Anhui 233000; \\ ${ }^{2}$ Key Laboratory of Cancer Prevention and Therapy Combining Traditional Chinese and Western Medicine, \\ Cancer Institute of Integrated Traditional Chinese and Western Medicine, Zhejiang Academy of Traditional Chinese Medicine, \\ Tongde Hospital of Zhejiang Province, Hangzhou, Zhejiang 310012; ${ }^{3}$ Department of Surgery, \\ The Second Affiliated Hospital of Jiaxing University, Jiaxing, Zhejiang 314000, P.R. China
}

Received March 15, 2020; Accepted July 30, 2020

DOI: $10.3892 / \mathrm{mmr} .2020 .11504$

\begin{abstract}
Pancreatic adenocarcinoma (PAAD) is a common and highly malignant tumor. The identification of prognostic biomarkers for PAAD could provide invaluable information for clinical treatment. AMP-activated protein kinase family member 5 (ARK5) is a member of the AMPK family that mediates the migration of PAAD cells. In the present study, ARK5 expression was evaluated using bioinformatics analysis in public datasets from The Cancer Genome Atlas. The expression levels of ARK5 in PAAD tumor tissue were significantly increased, compared with matched non-cancerous tissues. $A R K 5$ target genes were then predicted and Gene Ontology Biological Processes, Kyoto Encyclopedia of Genes and Genomes pathway analysis and Reactome gene sets were used to determine the functions associated with the target genes. A protein-protein interaction network was also constructed to find out the node genes and observe their association with the overall survival rate of PAAD. A total of nine node genes were identified in the PPI network, of which six were significantly upregulated in PAAD tissue, compared with matched normal tissue. The prognostic value of each node gene was evaluated by comparing the overall survival in patients with PAAD stratified according to the expression levels of these genes.
\end{abstract}

Correspondence to: Dr Zhengxiang Zhong, Faculty of Graduate Studies, Bengbu Medical College, 2600 Donghai Avenue, Bengbu, Anhui 233000, P.R. China

E-mail: 13505739209@139.com

Dr Xiaoguang Wang, Department of Surgery, The Second Affiliated Hospital of Jiaxing University, 397 Huancheng North Road, Jiaxing, Zhejiang 314000, P.R. China

E-mail: xiaoguangwangs@163.com

Key words: bioinformatics analysis, AMP-activated protein kinase family member 5 , biomarker, pancreatic adenocarcinoma, prognosis
Overall survival was significantly reduced in patients with high polo-like kinase-1 (PLK1) or protein phosphatase 1 catalytic subunit $\beta$ (PPPICB) expression, compared with patients with low expression of these genes. To further evaluate the relationship between PAAD and ARK5, ARK5 immunohistochemical staining was performed in a tissue microarray consisting of 112 tumor samples from patients with PAAD and adjacent normal tissue samples. ARK5 protein expression in PAAD tissue was markedly increased, compared with non-cancerous tissue $\left(\mathrm{P}=7.631 \times 10^{-11}\right)$. Moreover, ARK5 protein levels were associated with $\mathrm{N}$ stage $(\mathrm{P}=0.018)$. The overall survival of patients with PAAD with high ARK5 protein expression levels was reduced $(\mathrm{P}=0.014)$, compared with patients with low expression. In conclusion, these findings suggested that ARK5 may represent an independent prognostic indicator of PAAD.

\section{Introduction}

Pancreatic adenocarcinoma (PAAD) is a leading cause of cancer deaths worldwide. The prognosis of PAAD is poor, with the number of deaths almost matching the number of cases (1). Surgical resection is currently the only potentially curative option for PAAD. However, only $15-20 \%$ of patients present with a resectable tumor, and the 5-year survival rate following resection is only $4-5 \%(2,3)$. Moreover, the risk of local or distant recurrence in the first two years following resection is as high as $80 \%$ (4). Therefore, it is necessary to evaluate the patient survival rate following resection, yet current prediction methods are inadequate.

AMP-activated protein kinase family member 5 (ARK5) is a member of the AMPK family that mediates the migration of human PAAD cells (5). ARK5 activation is induced by Akt-dependent Ser600 phosphorylation (5). The Akt pathway is one of the key pathways that can mediate tumor progression by promoting the proliferation, survival and metastasis of cancer cells (6). Moreover, Akt signaling accelerates the progression of malignant tumors such as PAAD, breast cancer, colorectal cancer, squamous cell carcinoma and 
ovarian cancer (7-10). Moreover, tumor tissues often experience nutrient deficiency in their microenvironment (11), and ARK5 is the substrate of Akt during nutrient starvation $(5,12)$. ARK5 has been reported to promote tumor cell survival through Akt $(13,14)$.

The expression of the ARK5 gene is associated with PAAD metastasis (15). In hepatocellular carcinoma, breast cancer and colorectal cancer, patients with low expression levels of ARK5 display improved overall survival times compared with the high expression group (16-19). High $A R K 5$ expression in tumor tissue is associated with the metastatic potential of cancer, clinical stage and patient survival time $(16,20,21)$. Furthermore, a previous study demonstrated that ARK5 plays an essential role in cancer progression and chemotherapy resistance by inducing epithelial-mesenchymal transition (22). Therefore, it may be hypothesized that ARK5 is associated with PAAD prognosis and might play an active role in the progression of PAAD.

The aim of the present study was to analyze the expression levels of ARK5 using bioinformatics analysis of The Cancer Genome Atlas (TCGA) datasets. ARK5 target genes were identified, and their role in PAAD was examined. Lastly, in order to confirm whether ARK5 could represent an indicator of PAAD prognosis, ARK5 protein expression levels were determined in tumor samples from patients with PAAD and matched, normal, adjacent tissue.

\section{Materials and methods}

\section{TCGA database and bioinformatics analysis}

ARK5 expression in PAAD samples from TCGA. TCGA (https:// cancergenome.nih.gov/abouttcga/aboutdata/datalevelstypes) is a central repository of multidimensional experimental cancer data, comprising data pertaining to $>30$ types of human tumor. The expression profile of ARK 5 was obtained from different types of human cancer and corresponding non-cancerous tissue (Table I), including PAAD, and were analyzed using the Gene Expression Profiling Interactive Analysis (GEPIA) tool (v2.2019; http://gepia.cancer-pku.cn/) (23). GEPIA utilized the UCSC Xena (24) recomputed data of TCGA, and consulted with medical experts to determine the most appropriate sample grouping for tumor-normal comparisons. The datasets are stored in a MySQL relational database (version 5.7.17). Entering ARK5 in the 'General' field and clicking the 'GoPIA!': GEPIA generated the expression profile of ARK5.

Prediction and screening of target genes. Target genes (gene with a homology score $>0.5$ for $A R K 5$ ) (25) were predicted by entering ' $A R K 5$ ' into four databases; STRING v11.0 (https://string-db.org/), InBioMap v2019 (https://www.intomics. com/inbio/map), BioGRID v3.5.188 (https://thebiogrid.org/) and IntAct v4.2.15 (https://www.ebi.ac.uk/intact/). To improve the accuracy of prediction results, Venn diagrams were constructed and target genes overlapping between at least two of the four databases were selected for further analysis (http://bioinformatics.psb.ugent.be/webtools/Venn/).

Enrichment analysis of overlapping target genes. Enrichment analysis of overlapping target genes in ARK5 signaling was performed using Metascape v2019 (http://metascape. org/gp/index.html) (26). Gene Ontology (GO) Biological Processes, Kyoto Encyclopedia of Genes and Genomes (KEGG) Pathway and Reactome gene sets were used to determine the functions associated with the target genes.

Protein-protein interaction (PPI) network analysis of overlapping target genes. PPI analysis of ARK5 overlapping target genes was performed using Metascape and the node score was calculated and obtained in Metascape, with a node score cut-off of 1. Molecular Complex Detection (MCODE) algorithm, a module in Cytoscape v1.1 (27), was used to identify densely connected network neighborhoods, each MCODE component was labeled with a different color, and their biological significance are characterized.

Prognostic significance of the chosen node degree genes. Node genes were analyzed with the GEPIA tool to compare their expression levels varied in PAAD and adjacent normal tissues and whether these genes had any influence on the prognosis of PAAD. The gene symbol or gene ID (Ensembl ID) of node genes were entered in the 'Expression DIY' and 'Survival Analysis' fields respectively, and PAAD selected in the 'Dataset' field. Clicking the 'Plot' button caused GEPIA to present the gene expression box plot and survival plot of ARK5 in PAAD respectively.

\section{Validation experiments on PAAD clinical samples}

Patients and tissue collection. A PAAD tissue microarray (TMA) was obtained from the Biochip Shanghai National Engineering Research Center. All experimental procedures were approved by the Ethics Committee of Taizhou Hospital, authorizing TMA sample collection at Taizhou Hospital. The TMA included tumor tissue from 150 patients with PAAD who had undergone resection between January 2004 and December 2013. Of these, 112 included adjacent normal tissue. All patients signed an informed consent form. Clinicopathological data for all 150 patients included age, sex, grade, $\mathrm{T}$ stage, $\mathrm{N}$ stage, $\mathrm{M}$ stage, TNM stage, p53 expression and Ki67 expression. A total of 112 pairs of tumor samples were used and included adjacent normal tissues when analyzing the difference between cancer and normal tissues. In the correlation analysis of cancer and clinical data, 150 tumor samples were used, regardless of whether they contained adjacent normal tissues or not.

Immunohistochemical staining evaluation. PAAD tissue microarrays were immunohistochemically stained with Immunohistochemical kit $\left(\right.$ EnVision $^{\mathrm{TM}}$ FLEX+, cat. no. K8002, Dako; Agilent Technologies, Inc.) using an Automated Autostainer Link 48 system (Dako; Agilent Technologies, Inc.) by The Biochip Shanghai National Engineering Research Center.

To evaluate ARK5 protein expression, two pathologists scored the immunohistochemical staining using an Aperio scanner (Aperio XT, Leica Microsystems GmbH, magnification, $\mathrm{x} 200$ ); both were unaware of any clinical parameters. The cytoplasmic/nuclear staining intensity and ARK5 protein positive staining rate were determined using PAAD and corresponding non-cancerous tissues. The cytoplasmic and nuclear staining were scored separately. The staining intensity of each sample was given a modified Remmele score (26) that considers 
Table I. TCGA Datasets Evaluated (data from TCGA datasets).

\begin{tabular}{|c|c|c|c|}
\hline Types of cancer & TCGA dataset & No. of cancer tissues & No. of normal tissues \\
\hline Adrenocortical carcinoma & TCGA-ACC & 77 & 128 \\
\hline Bladder urothelial carcinoma & TCGA-BLCA & 404 & 28 \\
\hline Breast invasive carcinoma & TCGA-BRCA & 1,085 & 291 \\
\hline $\begin{array}{l}\text { Cervical squamous cell carcinoma and } \\
\text { endocervical adenocarcinoma }\end{array}$ & TCGA-CESC & 306 & 13 \\
\hline Cholangio carcinoma & TCGA-CHOL & 36 & 9 \\
\hline Colon adenocarcinoma & TCGA-COAD & 275 & 349 \\
\hline $\begin{array}{l}\text { Lymphoid neoplasm diffuse large B-cell } \\
\text { Lymphoma }\end{array}$ & TCGA-DLBC & 47 & 337 \\
\hline Esophageal carcinoma & TCGA-ESCA & 182 & 286 \\
\hline Glioblastoma multiforme & TCGA-GBM & 163 & 207 \\
\hline Head and neck squamous cell carcinoma & TCGA-HNSC & 519 & 44 \\
\hline Kidney chromophobe & TCGA-KICH & 66 & 53 \\
\hline Kidney renal clear cell carcinoma & TCGA-KIRC & 523 & 100 \\
\hline Kidney renal papillary cell carcinoma & TCGA-KIRP & 286 & 60 \\
\hline Acute myeloid leukemia & TCGA-LAML & 173 & 70 \\
\hline Brain lower grade glioma & TCGA-LGG & 518 & 207 \\
\hline Liver hepatocellular carcinoma & TCGA-LIHC & 369 & 160 \\
\hline Lung adenocarcinoma & TCGA-LUAD & 483 & 347 \\
\hline Lung squamous cell carcinoma & TCGA-LUSC & 486 & 338 \\
\hline Mesothelioma & TCGA-MESO & 87 & 0 \\
\hline Ovarian serous cystadenocarcinoma & TCGA-OV & 426 & 88 \\
\hline Pancreatic adenocarcinoma & TCGA-PAAD & 179 & 171 \\
\hline Pheochromocytoma and paraganglioma & TCGA-PCPG & 182 & 3 \\
\hline Prostate adenocarcinoma & TCGA-PRAD & 492 & 152 \\
\hline Rectum adenocarcinoma & TCGA-PEAD & 92 & 318 \\
\hline Sarcoma & TCGA-SARC & 262 & 2 \\
\hline Skin cutaneous melanoma & TCGA-SKCM & 461 & 558 \\
\hline Stomach adenocarcinoma & TCGA-STAD & 408 & 211 \\
\hline Testicular germ cell tumors & TCGA-TGCT & 137 & 165 \\
\hline Thyroid carcinoma & TCGA-THCA & 512 & 337 \\
\hline Thymoma & TCGA-THYM & 118 & 339 \\
\hline Uterine corpus endometrial carcinoma & TCGA-UCEC & 174 & 91 \\
\hline Uterine carcinosarcoma & TCGA-UCS & 57 & 78 \\
\hline
\end{tabular}

both the intensity and the percentage of cells stained at each intensity $(27,28)$. The staining intensity scores were defined as follows: i) 0 , negative; ii) 1 , weak; iii) 2 , strong; iv) 3 , very strong. The positive staining rate scores were defined as follows: i) 0 , negative; ii) $1,1-25 \%$; iii) $2,26-50 \%$; iv) $3,51-75 \%$; and v) 4 , $76-100 \%$. After multiplying the staining intensity score by positive staining rate score, patients were divided into low $(<6)$ and high $(\geq 6)$ expression groups according to the resultant scores.

Statistical analysis. ARK5 expression in PAAD samples and adjacent normal tissue was analyzed using $\chi^{2}$ tests and Fisher's exact test. The association between ARK5 protein expression and clinicopathological features was analyzed using a $\chi^{2}$ test or Fisher's exact test. A survival curve was constructed using the Kaplan-Meier method, and the log-rank statistical test was used for single-factor survival analysis. Statistical analysis was conducted using SPSS version 18.0 (SPSS, Inc.). $\mathrm{P}<0.05$ was considered to indicate a statistically significant difference.

\section{Results}

TCGA database and bioinformatics analysis ARK5 expression in PAAD samples from TCGA database. $A R K 5$ was overexpressed in several types of human cancer, including PAAD and head and neck squamous cell carcinoma. Moreover, ARK5 was also expressed at low levels in other tumors, such as cervical squamous cell carcinoma, endocervical adenocarcinoma, glioblastoma multiforme, brain low-grade glioma, lung adenocarcinoma, ovarian serous cystadenocarcinoma, skin cutaneous melanoma, uterine corpus endometrial carcinoma and uterine sarcoma $(\mathrm{P}<0.05)$. These results indicated that $A R K 5$ had different expression levels in different 
Table II. Pancreatic adenocarcinoma-associated genes identified in The Cancer Genome Atlas using four databases (data from TCGA datasets).

Database

InBio Map

STRING

BioGRID

IntAct
Gene name

BTRC, CDKN1A, CUL1, FBXO25, FBXW11, LATS1, PLK1, PPP1CB, PPP1CC, PPPIR12A, PPP1R12B, PPP1R12C, PRKAA1, SKP1, STK11, TP53, UBC, USP9X

AASS, ATM, CASP6, CCNA2, CCND1, CCND2, CCND3, CCNE1, CDK2, CDK4, CDKN1A, FBXW11, MDM2, MDM4, PPP1R12A, PPP1R12B, STK11, STRADA, TP53, USP9X

APP, BTRC, CRYM, CUL1, ELAVL1, FBXO25, FBXW11, FLT3, IGHA2, KIAA1429, KRT77, LATS1, PLK1, PPP1CB, PPP1CC, PPP1R12A, PPP1R12B, PPP1R12C, PRKAA1, PRKAR1A, PRPSAP1, S100A8, S100A9, SKP1, SRRM2, TNIP2, TP53, USP9X

BTRC, FBXW11, FYN, IGHA2, LATS1, PRKARIA, PRPSAP1, S100A8, S100A9, SRRM2, STK11, TP53, USP9X



Figure 1. ARK5 expression profile (data from TCGA datasets). ARK5 gene expression across tumor datasets and paired normal tissue datasets. Each dots represent expression of samples. According to the calculation of GEPIA tool, genes with higher log2FC values and higher percentage value than the thresholds are considered over-expressed genes. Red and green indicate high and low gene expression levels in tumor tissue, respectively. Black indicates that there were no differences in gene expression levels between tumor and normal tissue. ${ }^{*} \mathrm{P}<0.05$ vs. normal tissue.

tumors and is highly expressed in PAAD, in which it may exist as an oncogene (Fig. 1).

Prediction and screening of target genes. Using InBioMap, STRING, BioGRID, and IntAct, a total of 18, 20, 28, and 13 ARK5 candidate target genes were identified, respectively (Table II). Moreover, 23 genes were shared between at least two of the four databases and were therefore further selected as overlapping target genes for $A R K 5$. A Venn diagram illustrating the overlap between the four databases is illustrated in Fig. 2 .

Enrichment analysis of overlapping target genes in ARK5 signaling pathways. Pathway enrichment analysis was performed to determine the functions associated with the 23 target genes with respect to biological processes (Table III). The target genes of ARK5 participated in GO Biological Processes, such as 'cell cycle G2/M phase transition', 'regulation of circadian rhythm', 'negative regulation of cell cycle', 'intrinsic apoptotic signaling pathway', and 'protein destabilization' and in KEGG Pathways, including 'oxytocin signaling pathway' and 'FoxO signaling pathway', and Reactome gene sets including 'Signaling by TGF- $\beta$ Receptor complex'.

Identifying node degree genes via PPI network analyses of overlapping target genes. To explore the interaction between the 23 overlapping target genes, a PPI network was constructed 
Table III. Pathway enrichment analysis of overlapping target genes (data from TCGA datasets).

A, GO biological processes

\begin{tabular}{llccc}
\hline Term & \multicolumn{1}{c}{ Description } & Count & Frequency, $\%$ & $\log _{10}(\mathrm{P})$ \\
\hline GO:0044839 & Cell cycle G2/M phase transition & 11 & 47.83 & -15.46 \\
GO:0042752 & Regulation of circadian rhythm & 7 & 30.43 & -11.14 \\
GO:0045786 & Negative regulation of cell cycle & 9 & 39.13 & -10.97 \\
GO:0097193 & Intrinsic apoptotic signaling pathway & 6 & 26.09 & -8.47 \\
GO:0031648 & Protein destabilization & 3 & 13.04 & -6.63 \\
\hline
\end{tabular}

B, KEGG pathways

\begin{tabular}{llccc}
\hline Term & Description & Count & Frequency, $\%$ & $\log _{10}(\mathrm{P})$ \\
\hline hsa04921 & Oxytocin signaling pathway & 7 & 30.43 & -10.12 \\
hsa04068 & FoxO signaling pathway & 5 & 21.74 & -6.88 \\
\hline
\end{tabular}

C, Reactome gene sets

\begin{tabular}{|c|c|c|c|c|c|}
\hline Term & Description & Count & Frequency, \% & $\log _{10}(\mathrm{P})$ & $\log _{10}(q)$ \\
\hline R-HSA-170834 & Signaling by TGF-beta Receptor Complex & 3 & 13.04 & -4.35 & -2.24 \\
\hline
\end{tabular}

GO, Gene Ontology; KEGG, Kyoto Encyclopedia of Genes and Genomes; P, P-value; q, false positive rate calculated by P-value.

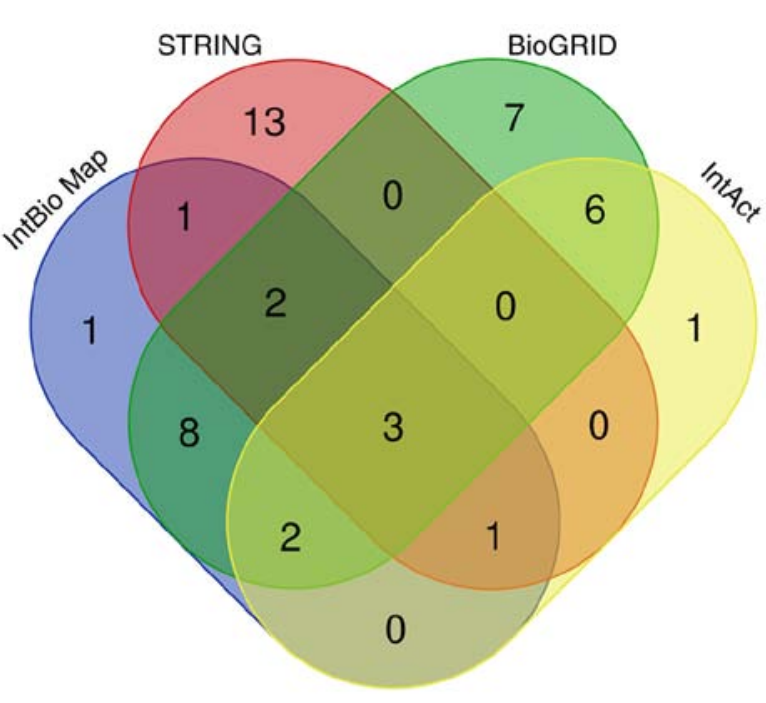

Figure 2. Venn diagram of predicted target genes from four databases (data from TCGA datasets). Different colors represent different databases.

using Metascape (Fig. 3). The Molecular Complex Detection (MCODE) algorithm was used to identify densely connected network neighborhoods, each MCODE component is labeled with a different color, and their biological significance are characterized [Red MCODE: MAP3K8 (TPL2)-dependent MAPK1/3 activation; Ubiquitin E3 ligase (SKP1A, BTRC, CUL1); Ubiquitin E3 ligase (FBXW11, SKP1, CUL1). Blue MCODE: PID PLK1 PATHWAY; Regulation of PLK1 Activity at G2/M Transition; G2/M Transition]. A total of 9 node

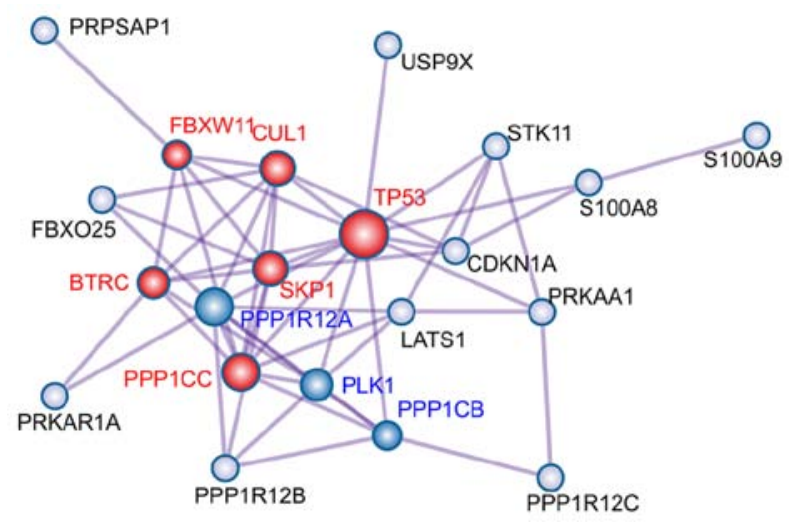

Figure 3. PPI network of overlapping target genes (data from TCGA datasets). A total of 23 genes were included in the PPI network. Each node represents a gene and proteins connected within a PPI network are likely to collaborate to perform various related biological processes. Red circles represent a node score of 2.5 , blue circles represent a node score of 1 , and white circles represent a node score of 0 (i.e., non-node genes). The Molecular Complex Detection algorithm was applied to identify densely connected network components, with a node score cut-off of 1 . PPI, protein-protein interaction.

genes were identified, including polo-like kinase 1 ( $P L K 1)$, protein phosphatase 1 catalytic subunit $\beta(P P P I C B)$, protein phosphatase 1 regulatory subunit 12A (PPPIR12A), tumor protein 53 (TP53); cullin 1 (CUL1); F-box and WD repeat domain-containing 11 ( $F B X W 11)$, S-phase kinase-associated protein 1 (SKP1), $\beta$-transducin repeat-containing E3 ubiquitin protein ligase $(B T R C)$ and protein phosphatase 1 catalytic subunit $\gamma(P P P I C C)$. 

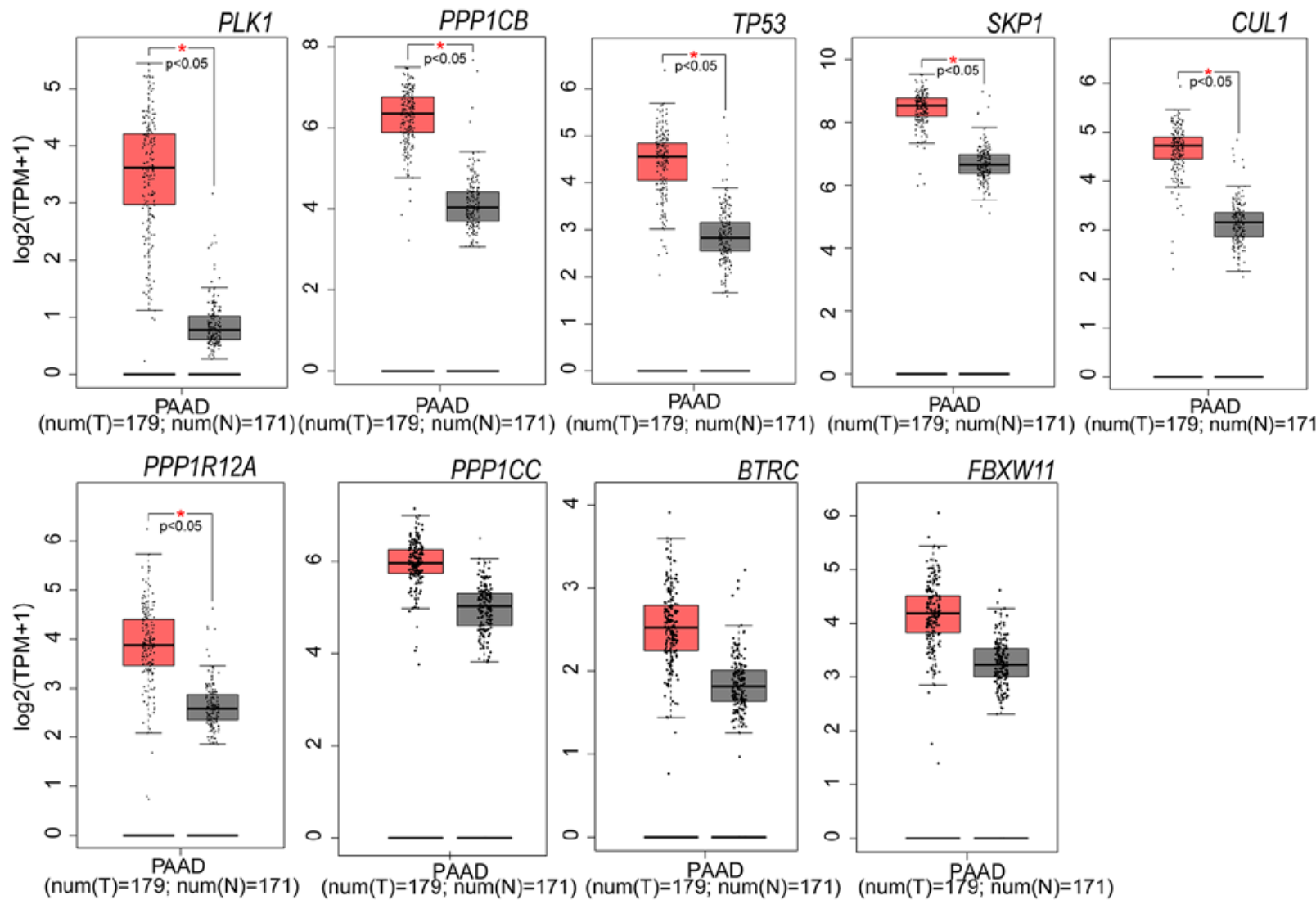

(num $(\mathrm{T})=179 ; \operatorname{num}(\mathrm{N})=171$ )

(num $(T)=179$; num

171) $(\operatorname{num}(\mathrm{T})=$ PAA; $\operatorname{num}(\mathrm{N})=171)$

Figure 4. Expression levels of nine selected genes in PAAD tissues and adjacent non-tumor tissues (The data were from TCGA datasets). Six of the nine genes are significantly upregulated in PAAD tissue $(\mathrm{n}=179)$, compared with adjacent non-tumor tissue $(\mathrm{n}=171)$. Red and grey represent tumor and normal adjacent tissues, respectively. ${ }^{*} \mathrm{P}<0.05$ vs. normal tissue. PAAD, pancreatic adenocarcinoma; $P L K 1$, polo-like kinase $1 ; P P P 1 C B$, protein phosphatase 1 catalytic subunit $\beta$; $P P P 1 R 12 A$, protein phosphatase 1 regulatory subunit 12A, TP53, tumor protein 53; $C U L 1$, cullin 1; FBXW11, F-box and WD repeat domain-containing $11 S K P 1$, S-phase kinase-associated protein $1 ; B T R C, \beta$-transducin repeat-containing E3 ubiquitin protein ligase; $P P P 1 C C$, protein phosphatase 1 catalytic subunit $\gamma$.

Prognostic significance evaluation of the nine node degree genes. The expression levels of the nine node genes were analyzed using the GEPIA tool in PAAD tissue and corresponding non-cancerous tissue. PLK1, PPP1CB, TP53, SKP1, $C U L 1$ and $P P P 1 R 12 A$ expression levels were significantly increased in PAAD tissue, compared with paired non-cancerous tissues (Fig. 4). To solve the imbalance between the tumor and normal data which can cause inefficiency in various differential analyses, the GEPIA tool downloaded the gene expression data that are re-computed from raw RNA-Seq data by the UCSC Xena (24) project based on a uniform pipeline. The GEPIA tool uses input from medical experts to determine the most appropriate sample grouping for tumor-normal comparisons. The datasets are stored in a MySQL relational database (version 5.7.17). In addition, the overall survival time of patients with PAAD and high expression $P L K 1$ or $P P P 1 C B$ was significantly reduced, compared with patients in the respective low-expression groups (Fig. 5).

\section{Validation experiments on PAAD clinical samples}

ARK5 protein expression levels in PAAD and corresponding non-cancerous tissues. ARK5 protein expression levels in 112 PAAD tissue and matched, adjacent, normal tissue were determined using immunohistochemistry (Fig. 6). ARK5 was highly expressed in $54(48.2 \%)$ and poorly expressed in $58(51.8 \%)$ of the 112 PAAD tissue samples. However, ARK5 protein was only highly expressed in $10(8.9 \%)$ and poorly expressed in $102(91.1 \%)$ normal tissue samples (Table IV). ARK5 protein expression levels were significantly higher in PAAD tissues, compared with paired non-cancerous tissue ( $\mathrm{P}<0.01$; Table IV).

ARK5 protein expression levels are associated with tumor $N$ stage. High ARK5 protein expression levels were associated with $\mathrm{N}$ stage $(\mathrm{P}=0.018)$. There were no significant associations with other clinicopathological characteristics, such as age or sex (Table V).

Association between ARK5 protein expression levels and overall survival. Kaplan-Meier survival analysis and the log-rank test were used for single-factor survival analysis. The overall survival time of patients with PAAD significantly differed between the high and the low ARK5 protein expression groups $(\mathrm{P}=0.014)$. Indeed, overall survival was significantly reduced patients with high ARK5 protein expression, compared with that in the low-expression group (Fig. 7), suggesting that ARK5 may be used as an independent prognosis factor for PAAD.

\section{Discussion}

PAAD is one of the leading causes of cancer deaths globally, particularly in developed nations. PAAD prognosis is generally very poor, with a 5-year survival rate at only 


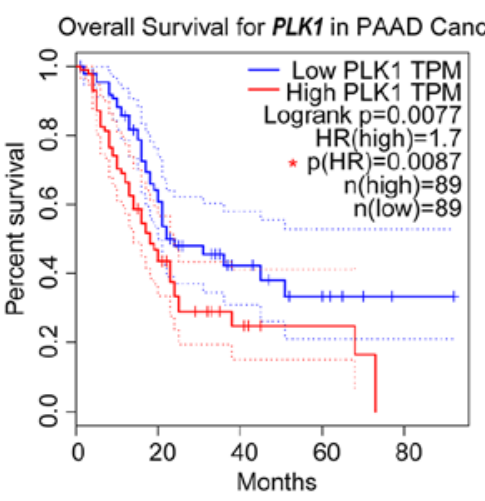

Overall Survival for SKP1 in PAAD Cancer

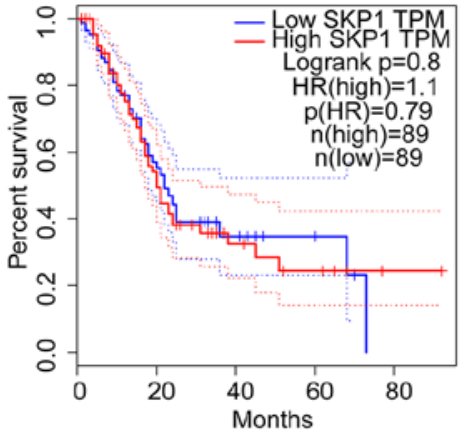

Overall Survival for PPP1CC in PAAD Cancer

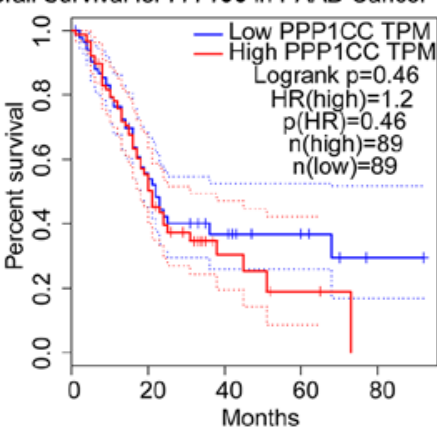

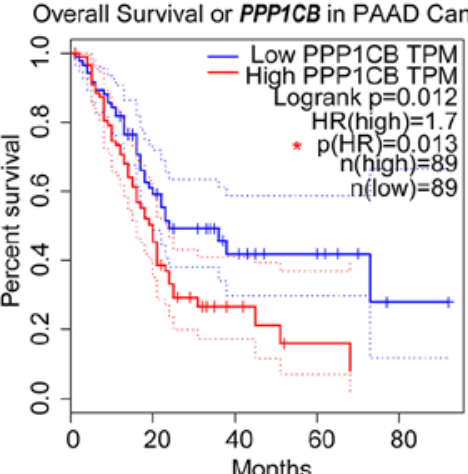

Overall Survival for CUL1 in PAAD Cancer

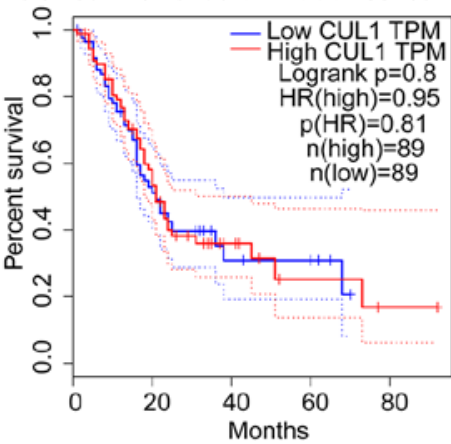

Overall Survival for BTRC in PAAD Cancer

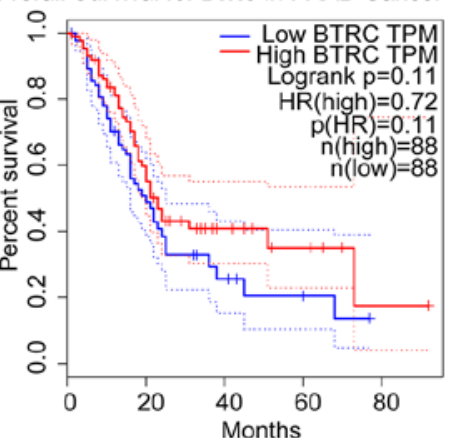

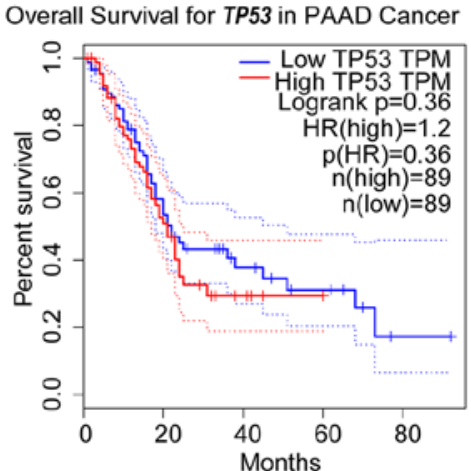

Overall Survival for PPP1R12A in PAAD Cancer

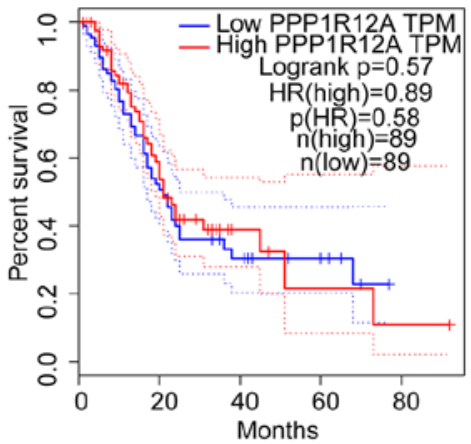

Overall Survival for FBXW11 in PAAD Cancer

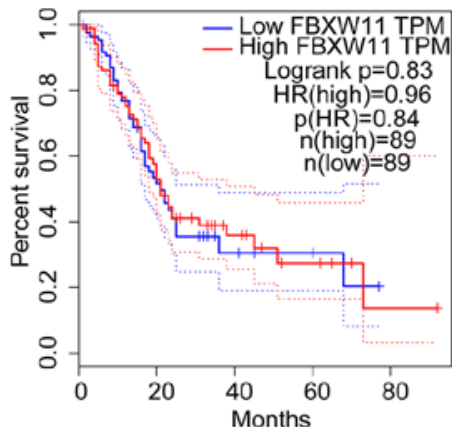

Figure 5. Prognostic information for the nine node genes. Online tools were used to generate Kaplan-Meier curves (data from TCGA datasets). The median value of the dataset was used as the cut-off for high and low expression for each gene. $P L K 1$ and $P P P 1 C B$ were associated with significantly worse survival rates. PAAD, pancreatic adenocarcinoma; $P L K 1$, polo-like kinase $1 ; P P P 1 C B$, protein phosphatase 1 catalytic subunit $\beta$; $P P P 1 R 12 A$, protein phosphatase 1 regulatory subunit $12 \mathrm{~A}, T P 53$, tumor protein 53; $C U L 1$, cullin 1; FBXW11, F-box and WD repeat domain-containing 11 SKP1, S-phase kinase-associated protein 1; BTRC, $\beta$-transducin repeat-containing E3 ubiquitin protein ligase; PPP1CC, protein phosphatase 1 catalytic subunit $\gamma$.

$\sim 4-5 \%$, and a postoperative recurrence rate within 2 years as high as $80 \%(1,3,4)$. Therefore, it is pivotal to identify an independent predictor for evaluating the prognosis of PAAD. Moreover, surgical resection is the only treatment that offers a potential cure of pancreatic cancer and conventional treatment methods, including chemotherapy and radiotherapy, only exist as auxiliary means (29). New therapies, such as tumor-specific targeted therapy, have emerged. Identifying novel targets is an integral part of tumor-specific targeted therapy (30).

ARK5 mediates the migration of human PAAD cells (5). A previous study identified an association between $A R K 5$ and gemcitabine resistance (22). In addition, ARK5 is reported to play an important role in tumor energy metabolism, providing a new class of potential target molecules for tumor therapy (31). In the present study, bioinformatics analysis was performed on TCGA datasets in order to determine whether ARK5 was associated with the prognosis of
Table IV. ARK5 protein expression levels in PAAD tissues are significantly upregulated compared with adjacent normal tissue (data from the clinical samples).

\begin{tabular}{lcrcr}
\hline & \multicolumn{2}{c}{ ARK5 expression } & & \\
\cline { 2 - 3 } Tissue type & High, $\mathrm{n}$ & Low, $\mathrm{n}$ & $\chi^{2}$ & P-value \\
\hline PAAD & 54 & 58 & 42.350 & $<0.01$ \\
Adjacent normal & 10 & 102 & & \\
\hline
\end{tabular}

$\mathrm{n}=112$ in each group. ARK5, AMP-activated protein kinase family member 5; PAAD, pancreatic adenocarcinoma.

PAAD, and the results were validated in clinical samples from patients with PAAD. 
A
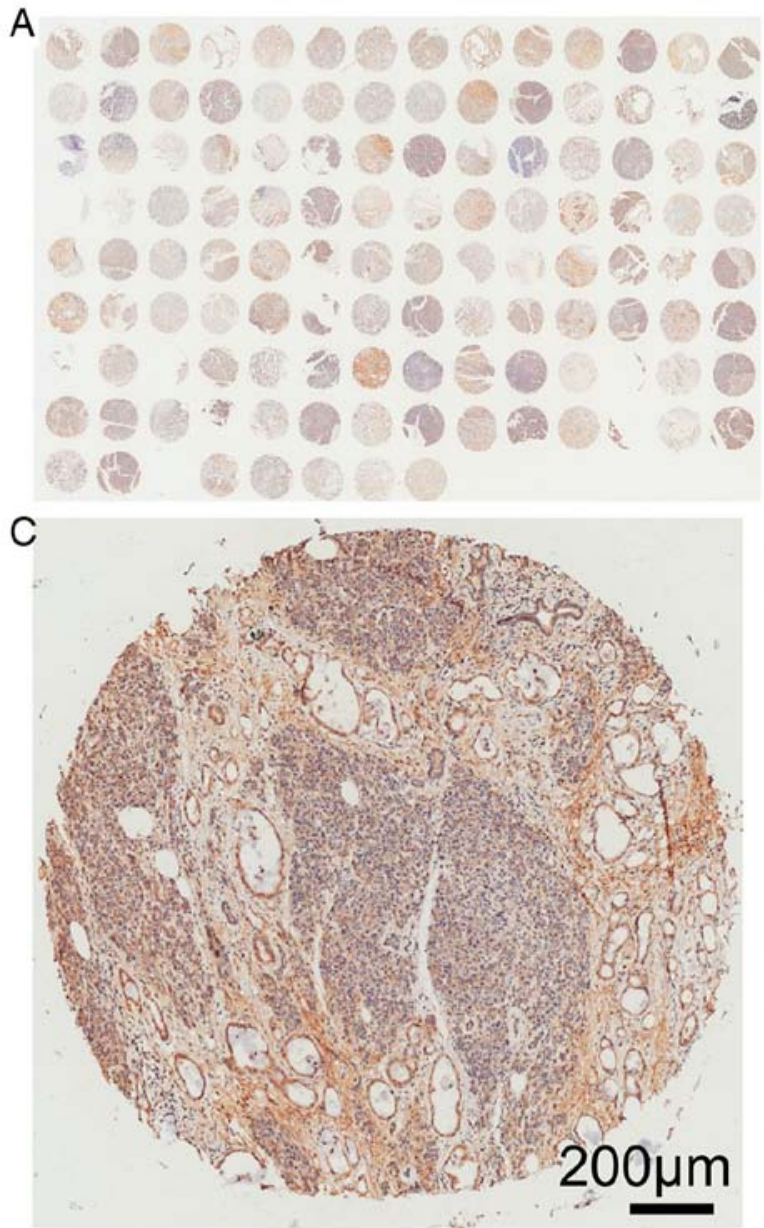

B
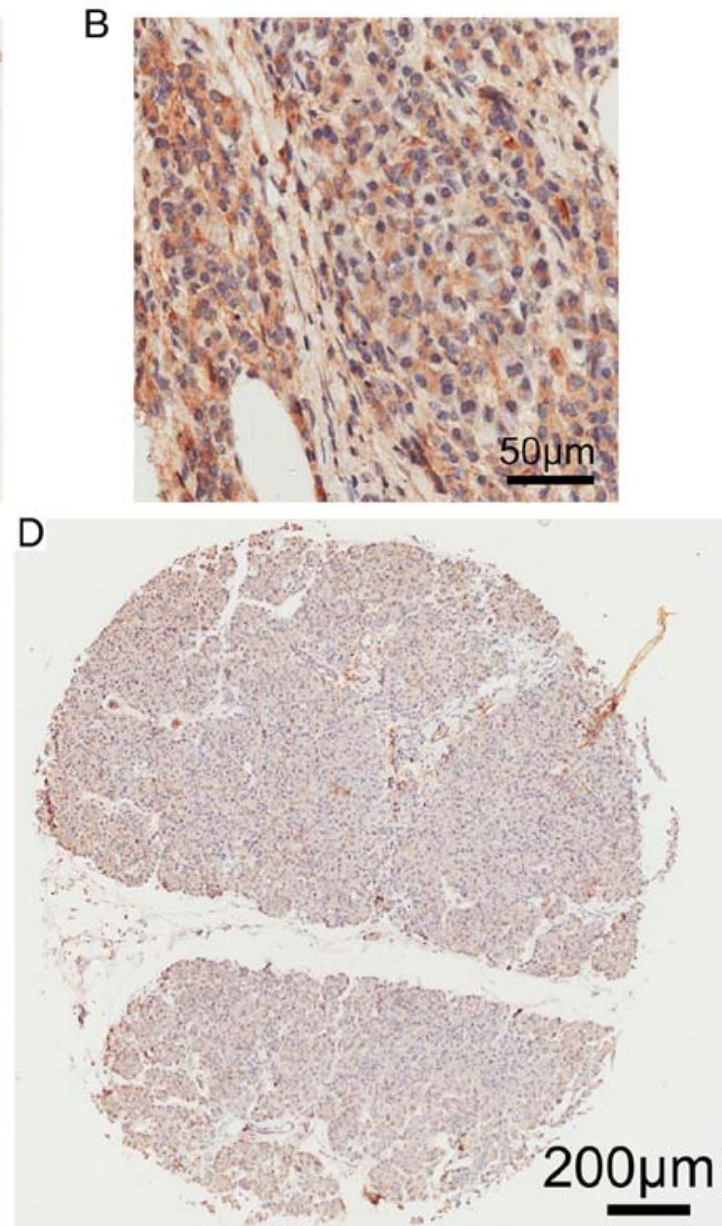

Figure 6. Photomicrographs of ARK5 immunohistochemical staining (data from the clinical samples). (A) Overview of the tissue array. (B) Positive staining for ARK5 in the cytoplasm of PAAD cells. (C) Positive staining for ARK5. (D) Negative staining for ARK5. ARK5, AMP-activated protein kinase family member 5 .



Figure 7. Kaplan-Meier analysis of the overall survival rate of patients with PAAD according to ARK5 protein expression levels (data from the clinical samples). ARK5 expression levels were determined using immunohistochemical staining in PAAD tissue microarrays. ARK5, AMP-activated protein kinase family member 5 ; PAAD, pancreatic adenocarcinoma.

In the present study, bioinformatics analysis results supported the hypothesis that $A R K 5$ served an active role in
PAAD development. ARK5 protein expression levels in tumor tissues were significantly higher than those in corresponding non-cancerous tissues. Additionally, elevated ARK5 protein expression levels were associated with tumor $\mathrm{N}$ stage.

Single-factor survival analysis indicated that high ARK5 protein expression levels in PAAD tissues were associated with worse overall survival rates. These findings indicated that ARK5 protein expression levels in PAAD tissues may prove useful when evaluating patient prognosis.

Although ARK5 is known to be associated with important pathways that drive the progression of PAAD and promote the proliferation, migration and invasion of PAAD cells, the prognostic significance of ARK5 for PAAD has not been thoroughly explored $(32,33)$. In conclusion, the present study suggested that ARK5 may represent an independent predictor for PAAD prognosis in a clinical setting. In addition, the present findings suggested that ARK5 might be used as a novel chemotherapeutic target for PAAD. However, these results, which are based on a Chinese cohort, should be further confirmed in other populations of patients with PAAD. In addition, due to the inevitable loss of clinicopathological data collection, there may be some errors in analyzing the association between ARK5 protein expression levels and clinicopathological characteristics, which is also be a limitation of the present study. 
Table V. Association between ARK5 protein expression levels and clinicopathological characteristics (data from the clinical samples).

\begin{tabular}{|c|c|c|c|c|c|}
\hline \multirow[b]{2}{*}{ Clinicopathological variable } & \multirow[b]{2}{*}{ Total cases } & \multicolumn{2}{|c|}{ ARK5 expression } & \multirow[b]{2}{*}{$\chi^{2}$} & \multirow[b]{2}{*}{ P-value } \\
\hline & & High, $n$ & Low, $\mathrm{n}$ & & \\
\hline \multicolumn{6}{|l|}{ Age (year) } \\
\hline$\leq 60$ & 65 & 32 & 33 & 0.009 & 0.926 \\
\hline$>60$ & 84 & 42 & 42 & & \\
\hline No data & 1 & & & & \\
\hline \multicolumn{6}{|l|}{ Sex } \\
\hline Female & 58 & 29 & 29 & $<0.01$ & $>0.999$ \\
\hline Male & 92 & 46 & 46 & & \\
\hline \multicolumn{6}{|l|}{ Grade } \\
\hline I & 4 & 0 & 4 & NA & $0.120^{\mathrm{a}}$ \\
\hline II/III & 146 & 75 & 71 & & \\
\hline \multicolumn{6}{|l|}{ T stage } \\
\hline $\mathrm{T} 1 / \mathrm{T} 2$ & 83 & 37 & 46 & 0.883 & 0.347 \\
\hline $\mathrm{T} 3$ & 53 & 28 & 25 & & \\
\hline No data & 14 & & & & \\
\hline \multicolumn{6}{|l|}{ N stage } \\
\hline N0 & 80 & 33 & 47 & 5.611 & 0.018 \\
\hline $\mathrm{N} 1$ & 62 & 38 & 24 & & \\
\hline No data & 8 & & & & \\
\hline \multicolumn{6}{|l|}{ M stage } \\
\hline M0 & 145 & 72 & 73 & NA & $>0.999^{\mathrm{a}}$ \\
\hline M1 & 5 & 3 & 2 & & \\
\hline \multicolumn{6}{|l|}{ TNM stage } \\
\hline I & 40 & 16 & 24 & 1.932 & 0.165 \\
\hline II/IV & 100 & 53 & 47 & & \\
\hline No data & 10 & & & & \\
\hline \multicolumn{6}{|l|}{ p53 } \\
\hline Negative & 33 & 17 & 16 & 2.879 & 0.090 \\
\hline Positive & 57 & 19 & 38 & & \\
\hline No data & 60 & & & & \\
\hline \multicolumn{6}{|l|}{ Ki67 } \\
\hline Negative & 25 & 12 & 13 & 0.634 & 0.426 \\
\hline Positive & 62 & 24 & 38 & & \\
\hline No data & 63 & & & & \\
\hline
\end{tabular}

${ }^{\text {a}}$ Fisher's exact test. ARK5, AMP-activated protein kinase family member 5; NA, not applicable.

\section{Acknowledgements}

Not applicable.

\section{Funding}

This work was supported by the Medical and Health Science and Technology Planning Project of Zhejiang Province (grant no. 2019KY219), the Science and Technology Planning Project of Jiaxing City (grant no. 2018AY32003), the Zhejiang Provincial Ten Thousand Plan for Young Top Talents (2018), the Training Objects of Health Innovative Talents of Zhejiang Health (2018), the Key Project Co-constructed by Zhejiang Province and the Ministry (grant no. WKJ-ZJ-1916), and the Natural Science Foundation of China (grant nos. 81972693, 81972674, and 31900543).

\section{Availability of data and materials}

All datasets used in the present study are available from the corresponding author on reasonable request. The datasets generated during the present study are available in the TCGA 
(https://cancergenome.nih.gov/abouttcga/aboutdata/datalevelstypes).

\section{Authors' contributions}

ZXZ, XGW and HKX made substantial contributions to conception and design. HKX was involved in drafting the manuscript. JYM, WC and HKX made substantial contributions to analysis and interpretation of data. XDY, FC, ZWS and JGF made substantial contributions to the use of TCGA website and the acquisition of bioinformatics data. All authors read and approved the final manuscript.

\section{Ethic approval and consent to participate}

All experimental procedures were approved by the Ethics Committee of Taizhou Hospital, Zhejiang Province, where sample collection took place. All samples were obtained through National Human Genetic Resources Sharing Service Platform. All patients signed an informed consent form.

\section{Patient consent for publication}

Not applicable.

\section{Competing interests}

The authors declare that they have no competing interests.

\section{References}

1. Bray F, Ferlay J, Soerjomataram I, Siegel RL, Torre LA and Jemal A: Global cancer statistics 2018: GLOBOCAN estimates of incidence and mortality worldwide for 36 cancers in 185 countries. CA Cancer J Clin 68: 394-424, 2018.

2. Hidalgo M, Cascinu S, Kleeff J, Labianca R, Löhr JM, Neoptolemos J, Real FX, Van Laethem JL and Heinemann V: Addressing the challenges of pancreatic cancer: Future directions for improving outcomes. Pancreatology 15: 8-18, 2015.

3. Maitra A and Hruban RH: Pancreatic cancer. Ann Rev Pathol 3: 157-188, 2008.

4. Javadi S, Karbasian N, Bhosale P, de Castro Faria S, Le O, Katz MH, Koay EJ and Tamm EP: Imaging findings of recurrent pancreatic cancer following resection. Abdom Radiol (NY) 43: 489-496, 2018.

5. Suzuki A, Kusakai G, Kishimoto A, Lu J, Ogura T, Lavin MF and Esumi $\mathrm{H}$ : Identification of a novel protein kinase mediating Akt survival signaling to the ATM protein. J Biol Chem 278: 48-53, 2003.

6. Warfel NA and Kraft AS: PIM kinase (and Akt) biology and signaling in tumors. Pharmacol Ther 151: 41-49, 2015.

7. Ekstrand AI, Jonsson M, Lindblom A, Borg A and Nilbert M: Frequent alterations of the PI3K/AKT/mTOR pathways in hereditary nonpolyposis colorectal cancer. Fam Cancer 9: $125-129,2010$.

8. Li B, Tsao SW, Li YY, Wang X, Ling MT, Wong YC, He QY and Cheung AL: Id-1 promotes tumorigenicity and metastasis of human esophageal cancer cells through activation of PI3K/AKT signaling pathway. Int J Cancer 125: 2576-2585, 2009.

9. Ohta T, Isobe M, Takahashi T, Saitoh-Sekiguchi M, Motoyama $\mathrm{T}$ and Kurachi H: The Akt and ERK activation by platinum-based chemotherapy in ovarian cancer is associated with favorable patient outcome. Anticancer Res 29: 4639-4647, 2009.
10. Simon PO Jr, McDunn JE, Kashiwagi H, Chang $K$, Goedegebuure PS, Hotchkiss RS and Hawkins WG: Targeting AKT with the proapoptotic peptide, TAT-CTMP: A novel strategy for the treatment of human pancreatic adenocarcinoma. Int J Cancer 125: 942-951, 2009.

11. Esumi H, Izuishi K, Kato K, Hashimoto K, Kurashima Y, Kishimoto A, Ogura T and Ozawa T: Hypoxia and nitric oxide treatment confer tolerance to glucose starvation in a 5'-AMP-activated protein kinase-dependent manner. J Biol Chem 277: 32791-3298, 2002.

12. Suzuki A, Kusakai G, Shimojo Y, Chen J, Ogura T, Kobayashi $M$ and Esumi $H$ : Involvement of transforming growth factor-beta 1 signaling in hypoxia-induced tolerance to glucose starvation. J Biol Chem 280: 31557-31563, 2005.

13. Suzuki A, Lu J, Kusakai G, Kishimoto A, Ogura T and Esumi H: ARK5 is a tumor invasion-associated factor downstream of Akt signaling. Mol Cell Biol 24: 3526-3535, 2004.

14. Suzuki A, Ogura T and Esumi H: NDR2 acts as the upstream kinase of ARK5 during insulin-like growth factor-1 signaling. J Biol Chem 281: 13915-21391, 2006.

15. Kusakai G, Suzuki A, Ogura T, Kaminishi M and Esumi H: Strong association of ARK5 with tumor invasion and metastasis. J ExpClin Cancer Res 23: 263-268, 2004.

16. Cui J, Yu Y, Lu GF, Liu C, Liu X, Xu YX and Zheng PY: Overexpression of ARK5 is associated with poor prognosis in hepatocellular carcinoma. Tumour Biol 34: 1913-1918, 2013.

17. Chang XZ, Yu J, Liu HY, Dong RH and Cao XC: ARK5 is associated with the invasive and metastatic potential of human breast cancer cells. J Cancer Res Clin Oncol 138: 247-254, 2012.

18. Sun X, Gao L, Chien HY, Li WC and Zhao J: The regulation and function of the NUAK family. J Mol Endocrinol 51: R15-R22, 2013.

19. Kusakai G, Suzuki A, Ogura T, Miyamoto S, Ochiai A, Kaminishi M and Esumi H: ARK5 expression in colorectal cancer and its implications for tumor progression. Am J Pathol 164: 987-995, 2004

20. Lu S, Niu N, Guo H, Tang J, Guo W, Liu Z, Shi L, Sun T, Zhou F, Li H, et al: ARK5 promotes glioma cell invasion, and its elevated expression is correlated with poor clinical outcome. Eur J Cancer 49: 752-763, 2013.

21. Chen P, Li K, Liang Y, Li L and Zhu X: High NUAK1 expression correlates with poor prognosis and involved in NSCLC cells migration and invasion. Exp Lung Res 39: 9-17, 2013.

22. Wang X, Song Z, Chen F, Yang X, Wu B, Xie S, Zheng X, Cai Y, Chen W and Zhong Z: AMPK-related kinase 5 (ARK5) enhances gemcitabine resistance in pancreatic carcinoma by inducing epithelial-mesenchymal transition. Am J Transl Res 10: 4095-4106, 2018.

23. Tang Z, Li C, Kang B, Gao G, Li C and Zhang Z: GEPIA: A web server for cancer and normal gene expression profiling and interactive analyses. Nucleic Acids Res 45: W98-W102, 2017.

24. Vivian J, Rao AA, Nothaft FA, Ketchum C, Armstrong J, Novak A, Pfeil J, Narkizian J, Deran AD, Musselman-Brown A, et al: Toil enables reproducible, open source, big biomedical data analyses. Nat Biotechnol 35: 314-316, 2017.

25. von Mering C, Jensen LJ, Snel B, Hooper SD, Krupp M, Foglierini M, Jouffre N, Huynen MA and Bork P: STRING: Known and predicted protein-protein associations, integrated and transferred across organisms. Nucleic Acids Res 33 (Database Issue): D433-D437, 2005.

26. Zhou Y, Zhou B, Pache L, Chang M, Khodabakhshi AH, Tanaseichuk O, Benner C and Chanda SK: Metascape provides a biologist-oriented resource for the analysis of systems-level datasets. Nat Commun 10: 1523, 2019.

27. Shannon P, Markiel A, Ozier O, Baliga NS, Wang JT, Ramage D, Amin N, Schwikowski B and Ideker T: Cytoscape: A software environment for integrated models of biomolecular interaction networks. Genome Res 13: 2498-2504, 2003.

28. Remmele W and Stegner HE: Recommendation for uniform definition of an immunoreactive score (IRS) for immunohistochemical estrogen receptor detection (ER-ICA) in breast cancer tissue. Pathologe 8: 138-140, 1987 (In German).

29. McGuigan A, Kelly P, Turkington RC, Jones C, Coleman HG and McCain RS: Pancreatic cancer: A review of clinical diagnosis, epidemiology, treatment and outcomes. World J Gastroenterol 24: 4846-4861, 2018. 
30. Liu H, Ma Q, Xu Q, Lei J, Li X, Wang Z and Wu E: Therapeutic potential of perineural invasion, hypoxia and desmoplasia in pancreatic cancer. Curr Pharm Des 18: 2395-2403, 2012.

31. Liu L, Ulbrich J, Müller J, Wüstefeld T, Aeberhard L, Kress TR, Muthalagu N, Rycak L, Rudalska R, Moll R, et al: Deregulated MYC expression induces dependence upon AMPK-related kinase 5. Nature 483: 608-612, 2012.

32. Rajamani D and Bhasin MK: Identification of key regulators of pancreatic cancer progression through multidimensional systems-level analysis. Genome Med 8: 38, 2016.
33. Huang X, Lv W, Zhang JH and Lu DL: miR-96 functions as a tumor suppressor gene by targeting NUAK1 in pancreatic cancer. Int J Mol Med 34: 1599-1605, 2014.

(i) (3) $९$ This work is licensed under a Creative Commons Attribution-NonCommercial-NoDerivatives 4.0 International (CC BY-NC-ND 4.0) License. 